\title{
The Effect of Oleanolic and Ursolic Acids on the Hemolytic Properties and Biofilm Formation of Listeria monocytogenes
}

\author{
ANNA KUREK ${ }^{1}$, KATARZYNA MARKOWSKA ${ }^{1}$, ANNA M. GRUDNIAK ${ }^{1}$, WIRGINIA JANISZOWSKA² \\ and KRYSTYNA I. WOLSKA ${ }^{1^{*}}$
}

\begin{abstract}
${ }^{1}$ Department of Bacterial Genetics, Institute of Microbiology, Faculty of Biology, University of Warsaw, Warsaw, Poland ${ }^{2}$ Department of Plant Biochemistry, Institute of Biochemistry, Faculty of Biology, University of Warsaw, Warsaw, Poland
\end{abstract}

Submitted 5 September 2013, revised 5 November 2013, accepted 16 November 2013

\begin{abstract}
Oleanolic acid and ursolic acid are pentacyclic triterpenoids isolated from a variety of medicinal plants, which have antibacterial activity. Listeria monocytogenes is a Gram-positive facultative pathogen, being the causative agent of listeriosis. The present study was carried out to evaluate the in vitro effect of sub-inhibitory concentrations of both triterpene acids on the pathogenicity determinants of L. monocytogenes: their hemolytic activity and biofilm forming ability. Oleanolic and ursolic acids inhibited listeriolysin $\mathrm{O}$ activity without influencing toxin secretion. Biofilm formation, and the viability of L. monocytogenes cells in biofilms was diminished by both compounds. Thus, both acids affected L. monocytogenes virulence. It was also demonstrated that oleanolic acid bound to the peptidoglycan of L. monocytogenes and this interaction was influenced by teichoic acids.
\end{abstract}

Ke y words: Listeria monocytogenes, biofilm, listeriolysin O, oleanolic acid, ursolic acid

\section{Introduction}

Compounds of therapeutic value extracted from medicinal plants are mostly secondary metabolites (Cowan, 1999). Oleanolic acid (OA) and ursolic acid (UA) are representatives of the pentacyclic triterpenoids whose structures are based on the isoprene moiety. Both compounds exhibit several pharmacological activities. The hepatoprotective, anti-inflammatory, antioxidant and anticancer activities of OA and UA are well documented (Ikeda et al., 2008; Pollier and Goossens, 2012). OA, UA and their derivatives also possess antibacterial activity, primarily against Gram-positive bacteria, including multidrug-resistant strains (Fontanay etal., 2008; Wolska etal., 2010). However, the broad application of OA/UA is currently restricted because their mechanism of action is still poorly understood and the side-effects on eukaryotic cells have not been fully characterized.

A relatively small number of studies have investigated the cellular targets and functions affected by OA/ UA. Ren and coworkers (2005) showed that UA caused differential gene expression in Escherichia coli and substantially inhibited biofilm formation by E. coli, Pseudomonas aeruginosa and Vibrio harveyi. Subsequently,
Chen and coworkers (2009) demonstrated the ability of the oleane-type triterpenoid, glycyrrhizin, to inhibit the diarrhea-inducing activity of the heat-labile enterotoxin produced by ETEC (enterotoxicogenic $\underline{E}$. $\underline{\text { coli }}$ ). The interaction between OA/UA and several other antibacterial agents - mainly conventional antibiotics - has been also reported (Ge et al., 2010). Of particular interest is their synergistic action with $\beta$-lactam antibiotics against Staphylococcus aureus and Staphylococcus epidermidis (Kurek et al., 2012) and the ability to induce stress response (Grudniak et al., 2011).

The Gram-positive pathogen Listeria monocytogenes is the causative agent of listeriosis, a disease that has increased markedly in Europe in recent years (Allerberger and Wagner, 2010). Listeriolysin O (LLO) is considered as a major virulence determinant of Listeria (Kayal and Charbit, 2006; Schnupf and Portnoy, 2007). Recent studies showed new roles for intracellular LLO, such as control of autophagy upon vacuolar escape, and revealed the extracellular activities of LLO, for example induction of bacterial entry into the cells and modulation of immune activity (Hamon etal., 2012). L. monocytogenes forms biofilms with a structure that varies greatly from one strain to another. Bacteria living in biofilms are less susceptible than planktonic cells to antimicrobial

\footnotetext{
* Corresponding author: K.I. Wolska, Department of Bacterial Genetics, Institute of Microbiology, Faculty of Biology, University of Warsaw, Miecznikowa St. 1, 02-096 Warsaw, Poland; phone: + 48225541 302, fax: + 48225541 402; e-mail: izabelaw@biol.uw.edu.pl
} 
agents, especially antibiotics (Renier et al., 2011). The factors responsible for this enhanced resistance include restricted penetration of antimicrobials into the biofilm structure, decreased growth rate and the expression of possible resistance genes (Lewis, 2001). For this reason, the ability to grow in a biofilm is known to enhance the pathogenicity of certain bacterial species.

It was previously proved that the cell wall constitutes a cellular target of OA/UA activity against the facultative pathogen Listeria monocytogenes. Both these pentacyclic triterpenoids affected morphology and enhanced autolysis of bacterial cells, influenced autolysis of the isolated cell wall, inhibited peptidoglycan turnover and quantitatively changed the profile of muropeptides obtained after the digestion of peptidoglycan with mutanolysin (Kurek etal., 2010). So far there is no available information on the ability of peptidoglycan to bind either OA or UA.

The aim of the present study was to gain some insight into the effect of OA/UA on L. monocytogenes virulence factors. The influence of these compounds on the secretion and activity of listeriolysin $\mathrm{O}$, biofilm formation and L. monocytogenes viability in biofilms was examined. As peptidoglycan is involved in L. monocytogenes biofilm formation, the ability of UA/OA to bind this compound was also studied.

\section{Experimental}

\section{Materials and Methods}

Bacterial strain, medium and reagents. Listeria monocytogenes (PCM 1291) was obtained from the Polish Culture Collection, Wrocław, Poland. The bacteria were grown in tryptic soy broth with yeast extract - TSYEB at $37^{\circ} \mathrm{C}$. OA, UA and all other reagents were purchased from Sigma. OA containing a tritium-labeled hydroxyl group $\left({ }^{3} \mathrm{H}-\mathrm{OA}, 1 \mathrm{mg} \mathrm{mL} \mathrm{m}^{-1}\right.$, activity $5.1 \mathrm{GBq}$ $\mathrm{mmol}^{-1}$ ) was provided by the Department of Plant Biochemistry, Faculty of Biology, University of Warsaw.

Measurement of the hemolytic activity of L. monocytogenes. An overnight culture of L.monocytogenes was grown in TSYEB medium with constant shaking. After dilution to attain an $\mathrm{A}_{600}$ of 0.1 , the cultures were incubated further to an $\mathrm{A}_{600}$ of 0.5 in medium supplemented with sub-inhibitory concentrations of OA or $\mathrm{UA}$ as required. Samples of $1 \mathrm{~mL}$ were centrifuged and $20 \mu \mathrm{L}$ aliquots of the supernatants were incubated with a $1 \%$ suspension of sheep red blood cells in phosphatebuffered saline (PBS), pH 6.5, in a total volume of $1 \mathrm{~mL}$ at $37^{\circ} \mathrm{C}$ for $30 \mathrm{~min}$. In the control set of experiments sheep red blood cells were incubated only with OA/UA in the conditions described above. After centrifugation, the supernatants were collected and their absorbance at $410 \mathrm{~nm}$ was measured. The extent of hemolysis was calculated as the percentage of the complete hemolysis achieved by lysing erythrocytes with $1 \%$ SDS.

Determination of the LLO concentration in culture supernatants. An overnight L.monocytogenes culture was diluted to an $\mathrm{A}_{600}$ of 0.1 and then divided into separate samples. One sample served as a control, while sub-inhibitory concentrations of OA or UA were added to others and incubation was continued to an $\mathrm{A}_{600}$ of 0.5 . Cells were harvested by centrifugation, and proteins from the supernatants were precipitated and concentrated using $10 \%$ tichloroacetic acid (TCA). The protein concentration in each sample was estimated using the bicinchoninic acid assay (Brown et al., 1989). Samples containing $5 \mu \mathrm{g}$ of precipitated protein were subjected to sodium dodecyl sulphate-polyacrylamide gel electrophoresis (SDS-PAGE) (Laemmli, 1970) and the resolved protein bands were transferred to polyvinylidene difluoride (PVDF) membranes. The membranes were incubated with rabbit anti-LLO polyclonal antibody (Abcam, UK, 1:1500 dilution) for $1 \mathrm{~h}$, washed, then incubated with goat anti-rabbit IgG - alkaline phosphatase conjugate, washed again and finally developed using 5-bromo-4-chloro-3-indolyl phosphate and nitro-blue tetrazolium (BCIP/NBT) in carbonate buffer as the chromogenic substrate. Images of the membranes were captured and analyzed using Image Master VDS (Fujifilm) and Image Master 1D Elite version 301 programs (NonLinear Dynamics, UK).

Quantitative determination of biofilm formation and measurement of cell viability in biofilms. Bacterial biofilms were developed in wells of polystyrene microtiter plates containing $200 \mu \mathrm{L}$ samples of culture diluted in TSYEB medium supplemented with $0.45 \%$ glucose and various amounts of the tested compounds. The lowest concentration of a compound that inhibited biofilm growth, as determined by crystal violet staining, was taken as the minimal biofilm inhibitory concentration (MBIC) (Smith et al., 2008). To determine the amount of biofilm in the wells of polystyrene microtiter plates, those formed after $24 \mathrm{~h}$ in the presence of OA, UA or with control samples were stained with crystal violet and, after brief washing with $0.85 \% \mathrm{NaCl}$, ethanol was added to each well to solubilize the crystal violet and the dye concentration in this wash was estimated by measuring the $\mathrm{A}_{570}$ with a microtiter plate reader (Sunrise, Tecan, Switzerland). To measure cell viability in biofilms, the 3-(4,5-dimethylthiazol-2-yl)-2,5-diphenyltetrazolium bromide (MTT) staining method was employed (Kairo et al., 1999) with minor modifications (Walencka et al., 2005). The intensity of the violet color of the resulting formazan was determined by measuring the $\mathrm{A}_{550}$ with a microtiter plate reader.

Measurement of the ability of $\mathrm{OA}$ to bind peptidoglycan. Peptidoglycan was isolated from L. monocyto- 
genes cells following the procedure described by Korsak and coworkers (2005). The final peptidoglycan preparations were divided into two parts: one part was lyophilized and the second was treated with 5\% TCA in order to remove teichoic acids. The prepared peptidoglycan samples were suspended in distilled water $\left(1 \mathrm{mg} \mathrm{mL}^{-1}\right)$ and amounts of $100 \mu \mathrm{g}$ were incubated with ${ }^{3} \mathrm{H}-\mathrm{OA}(0.05,0.25,0.5$ or $1 \mu \mathrm{g})$ for $30 \mathrm{~min}$ at room temperature. The binding reactions were terminated by adding a 10-fold excess of unlabeled OA followed by $5 \mathrm{~min}$ incubation. The samples were centrifuged $(16,000 \mathrm{~g}$, $10 \mathrm{~min}$ ), then the resulting pellets were washed with distilled water and analyzed using a scintillation counter. In the negative controls, the cell wall fragments in the binding reaction were replaced by water.

Statystical analysis. Data are shown as means of at least three experiments \pm SD. Statistical significance of the differences between experimental groups was calculated using two-tailed unpaired Student's test. $p$ value $<0.05$ was considered statistically significant.

\section{Results and Discussion}

The hemolytic activity of the supernatants of L. monocytogenes cultures treated with $0.5 \times$ or $0.75 \times$ minimal inhibitory concentration (MIC) of OA/UA and an untreated control culture was determined. MIC values of both compounds were $8 \mathrm{~g} \mathrm{~mL}^{-1}$ as determined previously (Kurek et al., 2012). Both triterpene acids inhibited LLO hemolytic activity. Compared with the control, the inhibition observed in cultures treated with $0.75 \times$ MIC of OA or UA amounted to 2.5 -fold and 2.8 -fold, respectively (Fig. 1). As it was demonstrated

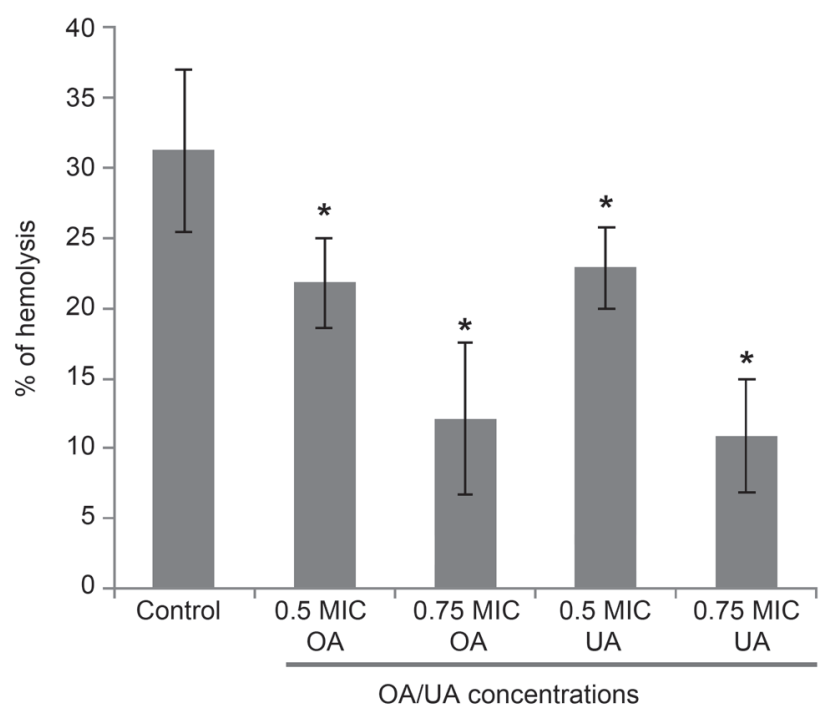

Fig. 1. Effect of OA and UA on LLO activity in L. monocytogenes culture supernatants. The results are the means of three independent experiments and the error bars indicate standard deviations $\left({ }^{*} \mathrm{p}<0.05\right.$ vs control).

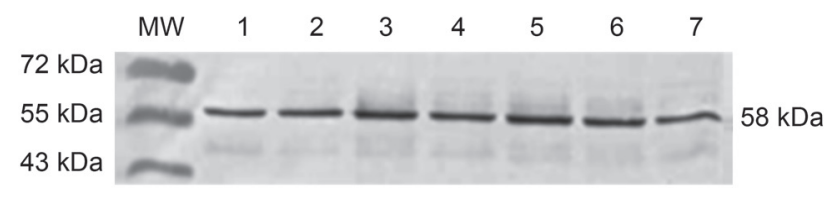

Fig. 2. Influence of OA and UA on LLO concentration in L. monocytogenes culture supernatants.

A representative Western blot is shown. MW - molecular weight standard; lane 1 - control culture without added OA/UA; lanes 2-4 - cultures treated with $0.25 \mathrm{MIC}, 0.5 \mathrm{MIC}$ and $0.75 \mathrm{MIC}$ of OA, respectively; lanes 5-7 - cultures treated with $0.25 \mathrm{MIC}, 0.5 \mathrm{MIC}$ and $0.75 \mathrm{MIC}$ of $\mathrm{UA}$, respectively.

that triterpenoids are able to form complexes with sterols (Osbourn, 1996) and cholesterol are present in erythrocyte membrane, the influence of OA/UA on erythrocytes in the absence of LLO was determined in the control experiments. It was shown that $\mathrm{OA}$ and $\mathrm{UA}$ in concentration $0.015 \times \mathrm{MIC}$, i.e. equivalent to $0.75 \times$ MIC present in LLO-containing supernatants (supernatants were diluted $50 \times$ in hemolytic activity assay, see Material and Methods) did not cause the lysis of erythrocytes. Analysis of SDS-PAGE followed by Western blotting demonstrated that the level of LLO in the culture supernatants was not diminished by treatment with either OA or UA (Fig. 2). The differences between densitometric measurement of peak areas did not exceeded $10 \%$. The synthesis of LLO was also not influenced by either of these compounds, since the amount of this cytolysin in whole cell lysates was unchanged by OA/UA treatment (data not shown). LLO is translated as a precursor polypeptide of 529 residues and its $\mathrm{N}$-terminal signal sequence is cleaved after protein secretion (Mengaud et al., 1988). The results of the present study indicate that OA and UA do not interfere with the functioning of the LLO secretion apparatus. Elucidation of the reason of the reduction of LLO hemolytic activity, for example by inhibiting initial binding to the erythrocyte membrane or subsequent step of pore formation, demands further experiments. In the control experiment the complexation capability of OA or UA with listeriolysin was checked in nondenaturing polyacrylamide gel shift assay (Mori et al., 2004) using $2.5 \mu \mathrm{g}$ of LLO and $0.08 \mu \mathrm{g}$ or $0.12 \mu \mathrm{g}$ OA or UA. No protein band shift was observed.

The ability of L. monocytogenes to form biofilms in the presence of OA/UA was estimated by crystal violet staining. OA and UA were added at $0.125,0.25$ or 0.5 minimal biofilm inhibitory concentration (MBIC). MBICs of both compounds were substantially higher than respective MICs $\left(24 \mu \mathrm{g} \mathrm{mL} \mathrm{m}^{-1}\right)$. Both compounds inhibited cell adhesion to the surface of polystyrene multiwell plates. Decreases in biofilm formation of about $15 \%$ and $>60 \%$ were observed in the presence of $0.25 \times \mathrm{MBIC}$ and $0.5 \times \mathrm{MBIC}$ of OA/UA, respectively. In the presence of $0.125 \times \mathrm{MBIC}$ of OA or UA, the biofilm biomass was decreased by only about $12.6 \%$ and $8.8 \%$, 
Table I

Influence of OA/UA on L. monocytogenes biofilm formation

\begin{tabular}{|l|c|c|}
\hline \multirow{2}{*}{$\begin{array}{c}\text { OA/UA concentration } \\
{[\mathrm{MBIC}]}\end{array}$} & \multicolumn{2}{|c|}{$\mathrm{A}_{570}$} \\
\cline { 2 - 3 } & $\mathrm{OA}$ & $\mathrm{UA}$ \\
\hline Control & $2.493 \pm 0.049$ & $2.493 \pm 0.049$ \\
\hline $0.125 \times \mathrm{MBIC}$ & $2.179 \pm 0.038^{\star}$ & $2.293 \pm 0.015$ \\
\hline $0.25 \times \mathrm{MBIC}$ & $2.097 \pm 0.042^{\star}$ & $2.175 \pm 0.021^{\star}$ \\
\hline $0.5 \times \mathrm{MBIC}$ & $0.984 \pm 0.037^{\star}$ & $0.861 \pm 0.032^{\star}$ \\
\hline
\end{tabular}

The results are the means of three independent experiments \pm standard deviation. MBICs of both OA and UA were $24 \mu \mathrm{g} \mathrm{mL} \mathrm{m}^{-1}$.

${ }^{*}$ Difference statistically significant $\left({ }^{*} \mathrm{p}<0.05\right.$ vs control).

respectively (Table I). The cell viability was reduced by $14 \%$ and $22 \%$ in the presence of $0.125 \times$ MBIC of OA or UA and by $42 \%$ and $56 \%$ in the presence of $0.5 \times$ MBIC of OA or UA, respectively (Fig. 3). Bacterial biofilms development is a multi-step process and their formation is influenced by a number of environmental factors including temperature, growth medium, $\mathrm{pH}$ and the nature of the surface (Moltz, 2005). Moreover, numerous molecular determinants are involved in the early stages of biofilm formation and the late stage of biofilm development among them is flagellum-mediated motility (O’Neil and Marquis, 2006). We previously observed that swarming-type, but not swimming-type nor twitching-type, motility of Pseudomonas aeruginosa was severely inhibited by OA and UA (data not shown), therefore it may be speculated that OA/UA can diminish L. monocytogenes biofilm formation by negatively influencing its swarming movement ability.

It has been also established that peptidoglycan plays an important role in L. monocytogenes biofilm formation. Previous results from our laboratory showed that peptidoglycan metabolism was affected by OA/UA

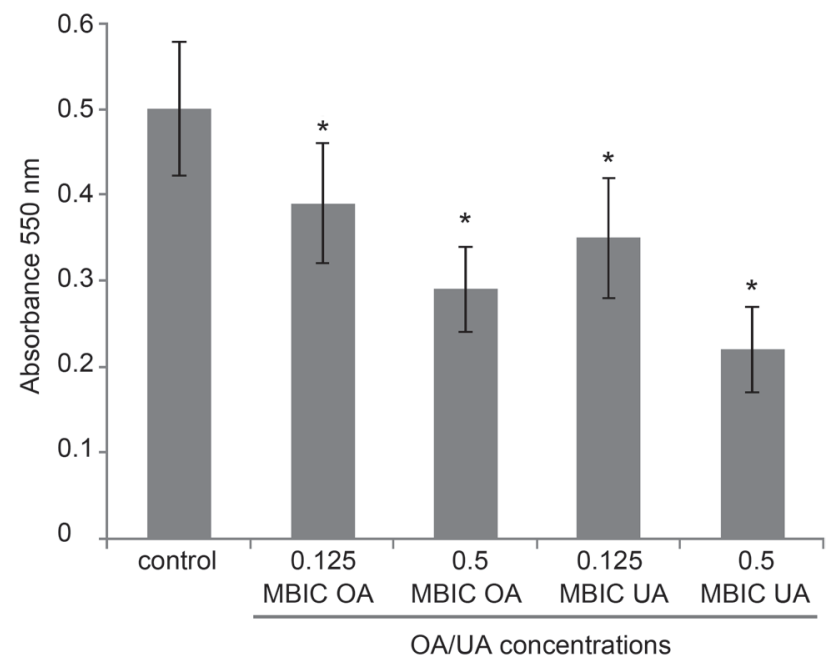

Fig. 3. Effect of OA and UA on the viability of L. monocytogenes cells in a biofilm. The results are the means of three independent experiments and the error bars indicate standard deviations $\left({ }^{*} \mathrm{p}<0.05\right.$ vs control).
Table II

Binding of radioactive OA to L. monocytogenes peptidoglycan

\begin{tabular}{|c|c|c|}
\hline \multirow{2}{*}{$\begin{array}{c}{ }^{3} \mathrm{H}-\mathrm{OA} \text { concentration } \\
{\left[\mu \mathrm{g} \mathrm{mL}^{-1}\right]}\end{array}$} & \multicolumn{2}{|c|}{$\begin{array}{l}\text { Radioactivity of peptidoglycan } \\
\text { (dpm per sample) }\end{array}$} \\
\hline & - Teichoic acids & + Teichoic acids \\
\hline $0.5^{*}$ & $322.52 \pm 55.32$ & $134.57 \pm 54.85$ \\
\hline $2.5^{\star}$ & $469.33 \pm 57.20$ & $148.11 \pm 46.84$ \\
\hline $5^{*}$ & $573.99 \pm 35.19$ & $320.02 \pm 143.40$ \\
\hline 10 & $875.92 \pm 31.12$ & $864.62 \pm 63.14$ \\
\hline
\end{tabular}

The presented values are the means of three independent experiments after subtraction of the background value \pm standard deviation. * Difference statistically significant $\left({ }^{*} \mathrm{p}<0.05\right)$.

(Kurek et al., 2010). In the present study we found that tritium-labeled OA bound to isolated peptidoglycan and that the teichoic acids, long anionic polymers threading through peptidoglycan layers that are crucial in protecting bacteria against harmful molecules and environmental stresses (Xia et al., 2010) only partially inhibited this binding (Table II). Taken together these results suggest that changes induced by OA and UA in the peptidoglycan structure of L. monocytogenes influence biofilm formation.

In conclusion, we have demonstrated that oleanolic and ursolic acids affect the virulence factors of L. monocytogenes. Both triterpene acids inhibit listeriolysin $\mathrm{O}$ activity without influencing its secretion and they also diminish the ability of this species to grow in biofilms.

\section{Acknowledgements}

This work was financially supported by Polish Ministry of Science and Higher Education grants no. N 304 11732/4332 and NN 302027937.

\section{Literature}

Allerberger F. and M. Wagner. 2010. Listeriosis: a resurgent foodborne infection. Clin. Microbiol. Infect. 16: 16-23.

Brown R.E., K.L. Jarvis and K.J. Hyland. 1989. Protein measurements using bicinchoninic acid: elimination of interfering substances. Anal. Biochem. 180: 136-139.

Chen J.C., T.Y. Ho, Y.S. Chang, S.L. Wu, C.C. Li and C.Y. Hsiang. 2009. Identification of Escherichia coli enterotoxin inhibitors from traditional medical herbs by in silico, in vitro and in vivo analyses. J. Ethnopharmacol. 121: 372-378.

Cowan M.M. 1999. Plant products as antimicrobial agents. Clin. Microbiol. Rev. 12: 564-582.

Fontanay S., M. Grare, J. Mayer, C. Finance and R.E. Duval. 2008. Ursolic, oleanolic and betulinic acids: Antibacterial spectra and selectivity indexes. J. Ethnopharmacol. 120: 272-276.

Ge F., F. Zeng, S. Liu, N. Guo, H. Ye, Y. Song, J. Fan, X. Wu, X. Deng, Q. Jin et al. 2010. In vitro synergistic interactions of oleanolic acid in combination with isoniazid, rifampicin or ethambutol against Mycobacterium tuberculosis. J. Med. Microbiol. 59: 567-572. Grudniak A.M., A. Kurek, J. Szarlak and K.I. Wolska. 2011 Oleanolic and ursolic acids affect the expression of the cysteine 
regulon and stress response in Escherichia coli. Curr. Microbiol. 62: 1331-1336.

Hamon M.A., D. Ribet, F. Stavru and P. Cossart. 2012. Listeriolysin O: the Swiss army knife of Listeria. Trends Microbiol. 20: 360-368.

Ikeda Y., A. Murakami and H. Ohigashi. 2008. Ursolic acid: an antiand proinflammatory triterpenoid. Mol. Nutr. Food Res. 52: 26-42. Kairo S.K., J. Bedwell, P.C. Tyler, A. Carter and M.J. Corbel. 1999. Development of tetrazolium salt assay for rapid determination of viability of BCG vaccines. Vaccine 17: 2423-2428.

Kayal S. and A. Charbit. 2006. Lysteriolysin O: a key protein of Listeria monocytogenes with multiple functions. FEMS Microbiol. Rev. 30: 514-529.

Korsak D., M. Popowska and Z. Markiewicz. 2005. Analysis of the murein of a Listeria monocytogenes EGD mutant lacking functional penicillin binding protein 5 (PBP5). Pol. J. Microbiol. 54: 339-342. Kurek A., A.M. Grudniak, M. Szwed, A. Klicka, Ł. Samluk and K.I. Wolska. 2010. Oleanolic acid and ursolic acid affect peptidoglycan metabolism in Listeria monocytogenes. Anton. Leeuven. Int. J. Gen. Mol. Microbiol. 97: 61-68.

Kurek A., P. Nadkowska, S. Pliszka and K.I. Wolska. 2012. Modulation of antibiotic resistance in bacterial pathogens by oleanolic acid and ursolic acid. Phytomedicine 19: 515-519.

Laemmli U.K. 1970. Cleavage of structural proteins during the assembly of the head of bacteriophage T4. Nature 227: 680-685.

Lewis K. 2001. Riddle of biofilm resistance. Antimicrob. Agents Chemother. 45: 999-1007.

Mengaud J., M.F. Vincente, J. Chenevert, J.M. Pereira, C. Geoffroy, B. Gicquel-Sanzey, F. Baquero, J.C. Perez-Diaz and P. Cossart. 1988. Expression in Escherichia coli and sequence analysis of the lysteriolysin $\mathrm{O}$ determinant of Listeria monocytogenes. Infect. Immun. 56: 766-772.

Moltz A.G. 2005. Formation of biofilms by Listeria monocytogenes under various growth conditions. J. Food Prot. 68: 92-97.
Mori M., M. Yoneyama, T. Ito, K. Takahashi, F. Inagaki and T. Fujita. 2004. Identification of Ser-386 of interferon regulatory factor 3 as critical target for inducible phosphorylation that determines activation. J. Biol. Chem. 279: 9698-9702.

O’Neil H.S. and H. Marquis. 2006. Listeria monocytogenes flagella are used for motility, not as adhesins, to increase host cell invasion. Infect. Immun. 74: 6675-6681.

Osbourn A.E. 1996. Preformed antimicrobial compounds and plant defense against fungal attack. Plant Cell 8: 1821-1831.

Pollier J. and A. Goossens. 2012. Oleanolic acid. Phytochemistry 77: $10-15$

Ren D., R. Zuo, A.F.G. Barrios, L.A. Bedzyk, G.R. Eldridge, M.E. Pasmore and T.K. Wood. 2005. Differential gene expression for investigation of Escherichia coli biofilm inhibition by plant extract ursolic acid. Appl. Environ. Microbiol. 71: 4022-4034.

Renier S., M. Hébraud and M. Desvaux. 2011. Molecular biology of surface colonization by Listeria monocytogenes: an additional facet of an opportunistic Gram-positive foodborne pathogen. Environ. Microbiol. 13: 835-850.

Schnupf P. and D.A. Portnoy. 2007. Listeriolysin O: a phagosomespecific lysin. Microb. Infect. 9: 1176-1187.

Smith K. A. Perez, G. Ramage, D. Lappin, C.G. Gemmell and S. Lang. 2008. Biofilm formation by Scottish clinical isolates of Staphylococcus aureus. J. Med. Microbiol. 57: 1018-1023.

Walencka E., B. Sadowska, S. Różalska, W. Hryniewicz and B. Różalska. 2005. Lysostaphin as a potential therapeutic agent for staphylococcal biofilm eradication. Pol. J. Microbiol. 54: 191-200.

Wolska K.I., A.M. Grudniak, B. Fiecek, A. Kraczkiewicz-Dowjat and A. Kurek. 2010. Antibacterial activity of oleanolic and ursolic acids and their derivatives. Centr. Eur. J. Biol. 5: 543-553.

Xia G., T. Kohler and A. Peschel. 2010. The wall teichoic acid and lipoteichoic acid polymers of Staphylococcus aureus. Int. J. Med. Microbiol. 300: 148-154. 
